\title{
Effect of Combined Systematized Behavioral Modification Education Program With Desmopressin in Patients With Nocturia: A Prospective, Multicenter, Randomized, and Parallel Study
}

\author{
Sung Yong Cho ${ }^{1}$, Kyu-Sung Lee ${ }^{2}$, Jang Hwan $\mathrm{Kim}^{3}$, Ju Tae Seo ${ }^{4}$, Myung-Soo Choo ${ }^{5}$, Joon Chul Kim ${ }^{6}$, Jong Bo Choi ${ }^{7}$, \\ Miho Song ${ }^{5}$, Ji-Youn Chun ${ }^{5}$, Seung-June $\mathrm{Oh}^{8}$ \\ ${ }^{1}$ Department of Urology, Seoul Metropolitan Government-Seoul National University Boramae Medical Center, Seoul National University College of Medicine, \\ Seoul, Korea \\ ${ }^{2}$ Department of Urology, Samsung Medical Center, Sungkyunkwan University School of Medicine, Seoul, Korea \\ ${ }^{3}$ Department of Urology, Severance Hospital, Yonsei University College of Medicine, Seoul, Korea \\ ${ }^{4}$ Department of Urology, Cheil General Hospital \& Women's Healthcare Center, Catholic Kwandong University College of Medicine, Seoul, Korea \\ ${ }^{5}$ Department of Urology, Asan Medical Center, University of Ulsan College of Medicine, Seoul, Korea \\ ${ }^{6}$ Department of Urology, Bucheon St. Mary's Hospital, The Catholic University of Korea College of Medicine, Bucheon, Korea \\ ${ }^{7}$ Department of Urology, Ajou University Hospital, Ajou University School of Medicine, Suwon, Korea \\ ${ }^{8}$ Department of Urology, Seoul National University Hospital, Seoul National University College of Medicine, Seoul, Korea
}

Purpose: The aims of this study were to investigate the efficacy of combining the systematized behavioral modification program (SBMP) with desmopressin therapy and to compare this with desmopressin monotherapy in the treatment of nocturnal polyuria (NPU).

Methods: Patients were randomized at 8 centers to receive desmopressin monotherapy (group A) or combination therapy, comprising desmopressin and the SBMP (group B). Nocturia was defined as an average of 2 or more nightly voids. The primary endpoint was a change in the mean number of nocturnal voids from baseline during the 3-month treatment period. The secondary endpoints were changes in the bladder diary parameters and questionnaires scores, and improvements in self-perception for nocturia.

Results: A total of 200 patients were screened and 76 were excluded from the study, because they failed the screening process. A total of 124 patients were randomized to receive treatment, with group A comprising 68 patients and group B comprising 56 patients. The patients' characteristics were similar between the groups. Nocturnal voids showed a greater decline in group B ( -1.5$)$ compared with group A (-1.2), a difference that was not statistically significant. Significant differences were observed between groups $\mathrm{A}$ and $\mathrm{B}$ with respect to the NPU index ( 0.37 vs. $0.29, \mathrm{P}=0.028)$, the change in the maximal bladder capacity $(-41.3 \mathrm{~mL}$ vs. $13.3 \mathrm{~mL}, \mathrm{P}<0.001)$, and the rate of patients lost to follow up $(10.3 \%$ [7/68] vs. $0 \%$ [0/56], $\mathrm{P}=0.016)$. Self-perception for nocturia significantly improved in both groups.

Conclusions: Combination treatment did not have any additional benefits in relation to reducing nocturnal voids in patients with NPU; however, combination therapy is helpful because it increases the maximal bladder capacity and decreases the NPI. Furthermore, combination therapy increased the persistence of desmopressin in patients with NPU.

Keywords: Nocturia; Polyuria; Behavior Therapy

- Fund Support: This study was supported by Ferring Pharmaceuticals research fund.

- Research Ethics: This trial was performed in accordance with the Declaration of Helsinki, and this study was approved by the Institutional Review Board of Seoul National University (IRB 06-2012-19). The ClinicalTrials.gov Identifier is NCT01576900. All patients provided written informed consent.

- Conflict of Interest: No potential conflict of interest relevant to this article was reported.

Corresponding author: Seung-June Oh (iD http://orcid.org/0000-0002-0322-3539 Department of Urology, Seoul National University Hospital, 101 Daehak-ro, Jongno-gu, Seoul 110-744, Korea

E-mail: sjo@snu.ac.kr / Tel: +82-2-2072-2406 / Fax: +82-2-742-4665

Submitted: November 9, 2014 / Accepted after revision: December 13, 2014
This is an Open Access article distributed under the terms of the Crecc
commons.org/licenses/by-nc/3.0/) which permits unrestricted non-commercial use, distribution, and reproduction in any medium, provided the original work is properly cited. 


\section{INTRODUCTION}

Nocturia severely affects a patient's quality of life (QoL) [1]. Nocturia is associated with many factors, including nocturnal polyuria (NPU), detrusor overactivity, excessive fluid intakes, alcohol or caffeine consumption in the evening, inappropriate sleep hygiene, and a shortage of daily exercise [2-4]. Medications such as desmopressin, alpha-blockers, or anticholinergic agents are usually recommended for patients with nocturia, and desmopressin is usually the first therapeutic choice. Behavioral therapy and medication are the initial treatment options for patients with overactive bladders [5]. Nocturia is one of several storage symptoms, and behavioral modification is considered the initial treatment option for patients with nocturia or NPU $[2,6]$. However, no standardized protocol has been established for the treatment of patients with NPU.

In a previous study, we demonstrated the efficacy of a systematized behavioral modification program (SBMP) in the absence of any medication [7]. We developed a 30-minute education program. In that study, we showed that education with 1 SBMP was sufficient for $78.5 \%$ of patients and that reinforcement education was helpful for an additional 7.6\% of patients with NPU. However, this SBMP has not been widely used in routine clinical practice, and no well-designed investigations have been undertaken that have examined the efficacy of combination therapy that comprises desmopressin and the SBMP.

The aims of the present study were to compare the efficacy of combination therapy, comprising desmopressin and the SBMP, with desmopressin monotherapy in patients with NPU, and to demonstrate the most important factors that determine improvements in the QoL of patients with NPU.

\section{MATERIALS AND METHODS}

\section{Patients}

Eligible patients included males and females who were aged 20 years or more and who had nocturia and NPU. Nocturia was defined as an average of 2 or more nightly voids and was determined using a 3-day frequency-volume chart. NPU was defined as a nocturnal urine volume that was more than one-third of the total daily urine volume. Patients who were excluded from this study included those with diabetes insipidus, polydipsia with hyponatremia with a serum sodium level of less than $135 \mathrm{mmol} /$ L, urinary incontinence, urinary tract infections, indwelling catheters, planned pregnancies, surgical histories involving the prostate gland or urethra, and patients taking drugs known or suspected to interact with desmopressin, including diuretics, tricyclic antidepressants, indomethacin, and carbamazepine. This trial was performed in accordance with the Declaration of Helsinki, and the Institutional Review Board of Seoul National University (IRB 06-2012-19) approved the study. The ClinicalTrials. gov Identifier is NCT01576900. All patients provided written informed consent.

\section{Study Design}

This was a prospective, multicenter, randomized parallel study that involved 8 centers. Using random number tables, patients were randomized $1: 1$ to receive $0.1 \mathrm{mg}$ or $0.2 \mathrm{mg}$ of desmopressin monotherapy or a combination of desmopressin therapy and the SBMP. In a previous study [8], the response within each treatment group was normally distributed with a standard deviation of 0.96 . We used the value from this study because there were no previously published studies available that had investigated differences between the SBMP and placebo-based behavioral therapy in patients with NPU with respect to nocturnal voids. The true difference in the nocturnal voids was 0.44 [8]. The probability of a type I error associated with testing this null hypothesis was 0.05 , and we needed to study 74 subjects in each group to be able to reject the null hypothesis that the mean number of nocturnal voids within the desmopressin monotherapy and the combined therapy groups was equal to a probability of 0.8 . The rate of patients lost to follow up was estimated at $25 \%$. The final sample size was determined to be 100 cases in each group.

The patients were advised to take desmopressin 1 hour before bedtime and to empty their bladders before they went to bed every night. Patients were told to avoid drinking more than 200 $\mathrm{mL}$ of water or other fluids. Any diuretics or caffeinated soft drinks were also prohibited in the evening. When a patient was assigned to the combination therapy group, desmopressin was prescribed and 1 SBMP was performed. The desmopressin was supplied by Ferring Pharmaceuticals (Saint-Prex, Switzerland).

The patients visited the outpatient clinic for their initial screening assessments on week 1 . The basic screening tests included assessing the patients' current illnesses, taking their medical histories, measuring their blood pressures, digital rectal examinations for men, and laboratory tests that included prostate-specific antigen levels and prostate ultrasonography for men, electrolyte chemistry, urinalysis with microscopy, urine culture, human chorionic gonadotropin levels in the urine for women, and uroflowmetry with postvoid urine volumes. Three-day frequency- 
volume charts recorded 24-hour fluid intakes and urine production, the times and volumes of the voids, bedtimes, and the times at which the patients rose. On week 3 , the second visit to the outpatient clinic occurred, which involved patient randomization and the prescription of desmopressin and SBMP education.

\section{Dose Titration}

All patients initially received $0.2-\mathrm{mg}$ desmopressin. Serum sodium levels were checked before the patients were randomized and at every visit to the clinic. The desmopressin dose was reduced from 0.2 to $0.1 \mathrm{mg}$ if sodium levels declined to less than $135 \mathrm{mmol} / \mathrm{L}$, or if the enrolled patients experienced intolerable adverse events (AEs). Desmopressin medication was stopped if the serum sodium level declined to less than $130 \mathrm{mmol} / \mathrm{L}$, or if the patients refused to take the medication.

\section{Behavioral Therapy}

Patients within the desmopressin monotherapy group were given brief explanations about the importance of fluid restriction after dinner during their appointments in the outpatient department. Patients within the combination therapy group underwent the SBMP that comprised a 30-minute education program [7]. During the education program, patients watched videos that explained the normal physiology underlying the process of urine formation and urination, and we explained the necessity of the behavioral modification program. We advised that $1,500 \mathrm{~mL}$ was the appropriate urine output volume, and that consuming caffeinated beverages or fluid-containing foods after dinner was prohibited. We provided specific guidance in relation to behavioral patterns. We emphasized the importance of sleep hygiene. Finally, we checked the patients' bladder diaries and gave feedback on these to improve the efficacy of the SBMP.

\section{Study Endpoints}

The primary endpoints were a change in the mean number of nocturnal voids and the proportion of $50 \%$ responders during the 3-month treatment period. A 50\% responder was defined as a patient who experienced a decline of at least $50 \%$ in the mean number of nocturnal voids from baseline to the time of the final visit to the outpatient clinic. The secondary endpoints were changes in the bladder diary parameters, International Consultation on Incontinence Questionnaire-Nocturia (ICIQ-N) scores, and nocturia quality-of-life (N-QoL) questionnaire scores, and improvements in self-perception for nocturia.

Patients completed the ICIQ-N and the N-QoL question- naire. Serum sodium levels were checked within 1 week of desmopressin being prescribed. The third and fourth visits to the outpatient clinic occurred after 1 and 3 months of treatment, respectively, following randomization. The patients completed 3-day frequency-volume charts, the ICIQ-N, the N-QoL questionnaire, the patient perception of bladder condition (PPBC) questionnaire, and the global response assessment (GRA). The ICIQ were linguistically validated by bilingual translators and were approved for clinical use by the International Consultation on Incontinence Modular Questionnaire Advisory Board (http:// www.iciq.net/index.html). A high level of overall linguistic validity has been demonstrated in a previous investigation in association with the N-QoL and the PPBC questionnaires [9].

The variables associated with the frequency-volume chart were the nocturnal urine volumes (NUV), maximal bladder capacity (MBC), nocturia index, actual number of nightly voids, nocturnal polyuria index (NPI), and the nocturnal bladder capacity index (NBCI).

\section{Safety and Tolerability}

Safety assessments were performed before the medication was prescribed and at the end of treatment, and they included the vital signs, physical examinations, body weights, and measurements of serum sodium levels. In the absence of any AE, patients were assigned to receive $0.2-\mathrm{mg}$ desmopressin. If the patients experienced any clinically significant $\mathrm{AE}$, such as voiding difficulties, peripheral edema, or sodium levels that were lower than 135 $\mathrm{mmol} / \mathrm{L}$, the desmopressin dose was reduced to $0.1 \mathrm{mg}$ or discontinued. All AEs were recorded at every clinic visit.

\section{Statistical Analysis}

Descriptive data, including the patients' characteristics, are presented. The treatment groups were compared in relation to the laboratory results and the clinical parameters using a stratified Cochran-Mantel-Haenszel test that controlled for age. A paired ttest was performed to determine whether any differences arose as a consequence of adding the SBMP to the management of patients who were being prescribed desmopressin. A P-value of $<0.05$ was considered statistically significant. All statistical analyses were performed using the SPSS ver. 17.0 (SPSS Inc., Chicago, IL, USA).

\section{RESULTS}

\section{Patient Characteristics}

In total, 200 patients (141 males and 59 females) were screened 


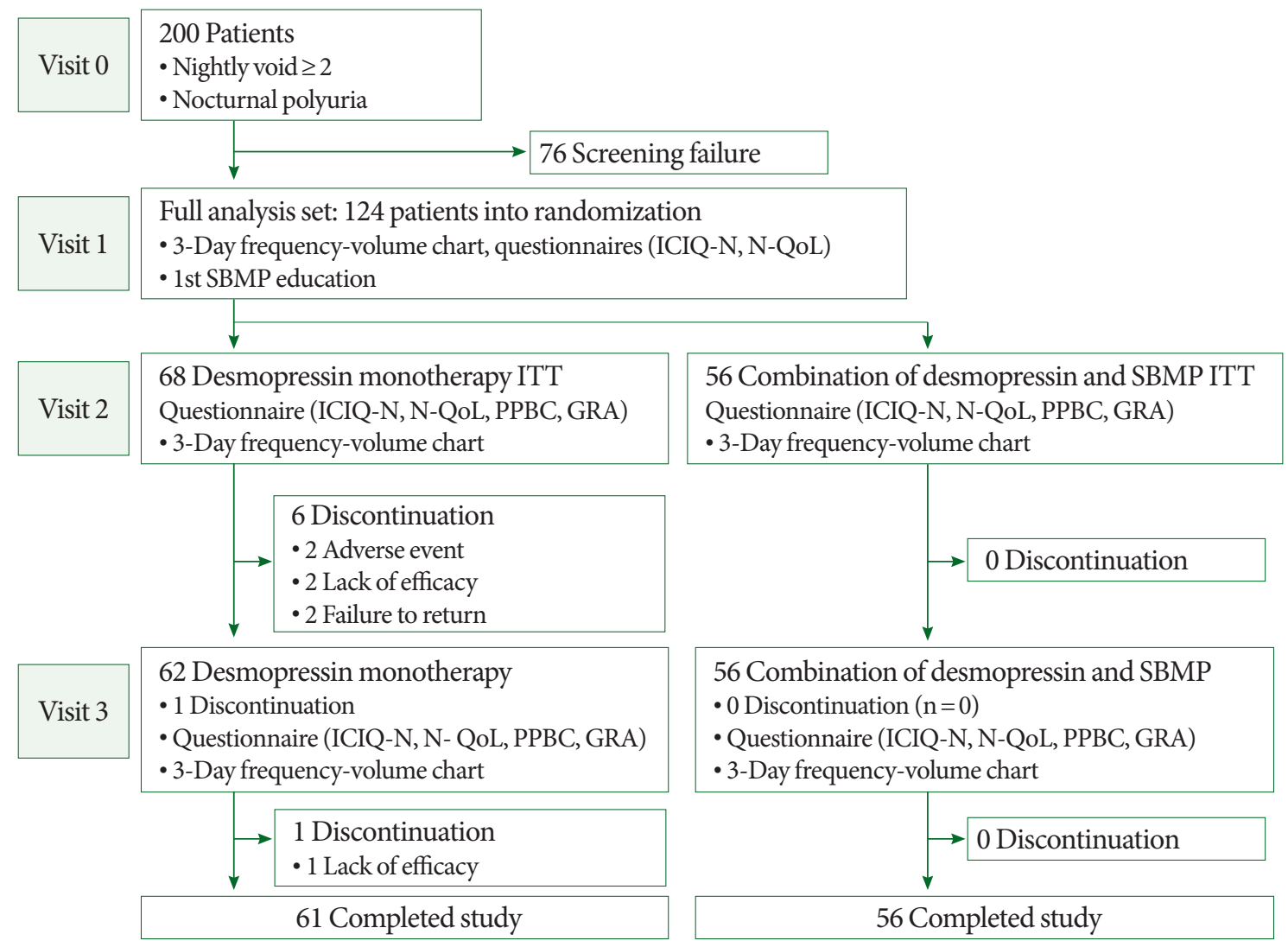

Fig. 1. Study design and disposition of patients. ICIQ-N, International Consultation on Incontinence Questionnaire-Nocturia; NQoL, nocturia quality of life; SBMP, systematized behavioral modification program; PPBC, patient perception of bladder condition; ITT, intention-to-treat; GRA, global response assessment.

to participate in the study at 8 centers. The mean ( \pm standard deviation) age of the patients was $66.8( \pm 8.5)$ years. A total of 76 patients were excluded from the study, because they fulfilled one or more of the exclusion criteria. As shown in Fig. 1, 124 patients entered the randomization and the dose-titration phases of the study, and they received at least 1 dose of desmopressin medication. Sixty-eight (54.8\%) and 56 patients (45.2\%) were randomized to receive either desmopressin monotherapy (group A) or combined therapy with desmopressin and the SBMP (group B), respectively. Seven patients in the desmopressin monotherapy group and none of the patients in the combined therapy group stopped the medication during the study period. The patients' characteristics were similar in both study groups (Table 1), and the study completion rate was $89.7 \%$ for group $A$ and $100 \%$ for group $B(P=0.016)$. A significantly larger $\mathrm{MBC}$ was evident in the desmopressin monotherapy group compared with the combination therapy group.

\section{Primary Endpoints}

Nocturia improved significantly in both treatment groups. Nocturnal voids significantly declined from baseline to the end of the study $(-1.32 \pm 1.39, \mathrm{P}<0.001)$. The combination therapy group showed a greater decline in the number of nocturnal voids compared with the desmopressin monotherapy group, and the proportion of patients with a 50\% reduction in the mean number of nocturnal voids was higher in the combination therapy group than in the desmopressin monotherapy group (Table 2). However, no statistically significant differences were evident between the treatment groups in relation to the number of nocturnal voids or the clinical response rates. There were no differences between the sexes with respect to the number of nocturnal voids or the clinical response rates.

\section{Secondary Endpoints}

Changes in the N-QoL questionnaire scores showed that the scores associated with 'a change of productivity the next day' 
Table 1. Patient demographics and baseline characteristics

\begin{tabular}{|c|c|c|c|}
\hline \multirow{2}{*}{ Variable } & \multicolumn{2}{|c|}{ ITT population } & \multirow{2}{*}{ P-value } \\
\hline & Desmopressin $(n=68)$ & Combination $(\mathrm{n}=56)$ & \\
\hline Male & $51(75.0)$ & $44(78.6)$ & 0.675 \\
\hline Age (yr) & $67.0 \pm 8.2(39.0-81.0)$ & $66.5 \pm 8.9(41.0-85.0)$ & 0.738 \\
\hline Height $(\mathrm{cm})$ & $165.4 \pm 7.9(148.0-180.0)$ & $164.3 \pm 8.1(143.0-183.0)$ & 0.478 \\
\hline Body weight (kg) & $65.8 \pm 70.5(37.0-93.0)$ & $65.3 \pm 9.7(42.2-94.0)$ & 0.790 \\
\hline Blood pressure_systolic (mm Hg) & $124.3 \pm 11.0(95.0-150.0)$ & $122.9 \pm 10.2(91.0-149.0)$ & 0.454 \\
\hline Blood pressure_diastolic (mm Hg) & $75.5 \pm 7.8(51.0-92.0)$ & $77.7 \pm 7.9(54.0-97.0)$ & 0.113 \\
\hline \multicolumn{4}{|l|}{ Comorbidity } \\
\hline Hypertension & $29(42.6)$ & $22(39.3)$ & 0.718 \\
\hline Diabetes mellitus & $15(22.1)$ & $11(19.6)$ & 0.826 \\
\hline \multicolumn{4}{|l|}{ Uroflowmetric parameters } \\
\hline Maximal urine flow $(\mathrm{mL} / \mathrm{sec})$ & $14.7 \pm 7.4(5.3-39.7)$ & $14.1 \pm 8.2(5.1-45.1)$ & 0.678 \\
\hline Voided urine volume (mL) & $188.5 \pm 113.2(47.7-582.0)$ & $168.1 \pm 88.3(36.0-377.6)$ & 0.331 \\
\hline Postvoid residual urine $(\mathrm{mL})$ & $14.3 \pm 19.5(0-89.0)$ & $13.7 \pm 22.3(0-99.0)$ & 0.889 \\
\hline \multicolumn{4}{|l|}{ N-QoL questionnaires } \\
\hline Total & $44.4 \pm 8.1(21.0-60.0)$ & $41.5 \pm 9.0(23.0-61.0)$ & 0.062 \\
\hline Subscores (sleep/energy) & $22.1 \pm 5.3(6-30)$ & $20.5 \pm 6.2(6.0-40.0)$ & 0.141 \\
\hline Subscores (bother/concern) & $21.8 \pm 5.0(11.0-34.0)$ & $20.3 \pm 5.5(11.0-33.0)$ & 0.112 \\
\hline \multicolumn{4}{|l|}{ Voiding diary parameters } \\
\hline 24-Hour total urine volume $(\mathrm{mL})$ & $1,922.6 \pm 901.0(870-6,450)$ & $1,888.4 \pm 976.0(567-6,510)$ & 0.841 \\
\hline Nocturnal urine volume (mL) & $856.8 \pm 500.4(345-3,570)$ & $831.9 \pm 404.6(200-2,850)$ & 0.767 \\
\hline Total number of nightly voids & $2.5 \pm 1.1(5-21)$ & $2.8 \pm 1.2(6-80)$ & 0.141 \\
\hline Maximal bladder capacity $(\mathrm{mL})$ & $360.3 \pm 105.2(130-550)$ & $319.7 \pm 105.1(100-550)$ & $0.036^{*}$ \\
\hline Nocturia index & $2.4 \pm 0.9(0.7-6.6)$ & $3.0 \pm 2.7(1.3-20.0)$ & 0.072 \\
\hline Nocturnal polyuria index & $0.4 \pm 0.1(0.2-0.7)$ & $1.3 \pm 5.4(0.1-40.0)$ & 0.242 \\
\hline Nocturnal bladder capacity index & $0.8 \pm 0.8(0-3.7)$ & $0.7 \pm 0.7(0-3.0)$ & 0.429 \\
\hline
\end{tabular}

Values are presented as number (\%) or mean \pm standard deviation (range). ITT, intention-to-treat; N-QoL, nocturia quality of life.

${ }^{\star} \mathrm{P}<0.05$, statistically significant.

(question 4) and 'concern of disturbing others in the house' (question 8) were more significantly affected in the combination therapy group compared with the desmopressin monotherapy group. Self-perceived improvements were demonstrated in the PPBC and GRA scores; however, no statistically significant differences were observed between the treatment groups with respect to the PPBC and GRA scores. The bladder diary parameters showed that only the NPI showed a significant difference between the treatment groups. When we analyzed the changes in each bladder diary parameter from the beginning of the study to the final visit to the outpatient clinic at the end of the study, a significant difference was observed between the desmopressin monotherapy group and the combination therapy group in relation to the change in the $\mathrm{MBC}(-41.3 \pm 96.3 \mathrm{~mL}$ vs. $13.3 \pm 37.9$ $\mathrm{mL} ; \mathrm{P}<0.001)$. None of these results showed any differences according to sex.

The SBMP increased the study completion rate in patients with nocturia. The rate of patients lost to follow up was 10.3\% (7/68) and $0 \%(0 / 56)$ in the desmopressin monotherapy group and the combination therapy group, respectively $(\mathrm{P}=0.016)$. 
Table 2. Responses to desmopressin and combination treatments

\begin{tabular}{|c|c|c|c|}
\hline \multirow{2}{*}{ Variable } & \multicolumn{2}{|c|}{ ITT population } & \multirow{2}{*}{ P-value } \\
\hline & Desmopressin $(n=68)$ & Combination $(\mathrm{n}=56)$ & \\
\hline \multicolumn{4}{|l|}{ Primary endpoints } \\
\hline Nocturnal voids & $-1.18 \pm 1.37(-3.0$ to 1.0$)$ & $-1.48 \pm 1.41(-5$ to 1$)$ & 0.227 \\
\hline \multicolumn{4}{|l|}{ Change in N-QoL questionnaires scores } \\
\hline 1. Difficult to concentrate the next day & $0.89 \pm 1.29(-3$ to 4$)$ & $0.69 \pm 1.17(-3$ to 4$)$ & 0.354 \\
\hline 2. Feel low in energy the next day & $0.80 \pm 1.19(-2$ to 4$)$ & $0.69 \pm 1.13(-3$ to 4$)$ & 0.592 \\
\hline 3. Nap during the day & $0.64 \pm 1.07(-2$ to 3$)$ & $0.45 \pm 1.16(-2$ to 3$)$ & 0.337 \\
\hline 4. Less productive the next day & $1.00 \pm 1.32(-3$ to 3$)$ & $0.46 \pm 1.05(-2$ to 4$)$ & $0.014^{*}$ \\
\hline 5. Participate less in activities I enjoy & $0.57 \pm 1.36(-3$ to 2$)$ & $0.36 \pm 0.95(-3$ to 4$)$ & 0.325 \\
\hline 6. Careful about when or how much I drink & $0.20 \pm 1.52(-2$ to 3$)$ & $0.31 \pm 1.12(-4$ to 4$)$ & 0.624 \\
\hline 7. Difficult to get enough sleep at night & $1.45 \pm 2.41(-2$ to 4$)$ & $1.32 \pm 1.48(-13$ to 4$)$ & 0.720 \\
\hline 8. Disturbing others in the house & $1.30 \pm 1.55(-4$ to 4$)$ & $0.45 \pm 1.42(-2$ to 4$)$ & $0.002^{\star}$ \\
\hline 9. Preoccupied about having to get up & $1.34 \pm 1.50(-1$ to 4$)$ & $1.21 \pm 1.39(-2$ to 4$)$ & 0.617 \\
\hline 10. Worried that this condition will get worse & $1.39 \pm 1.46(-2$ to 4$)$ & $1.36 \pm 1.41(-1$ to 4$)$ & 0.894 \\
\hline 11. Worry about no effective treatment & $1.11 \pm 1.64(-3$ to 4$)$ & $1.15 \pm 1.57(-3$ to 4$)$ & 0.885 \\
\hline 12. How bothersome has getting up at night & $-1.51 \pm 1.48(-5$ to 2$)$ & $-1.61 \pm 1.42(-4$ to 2$)$ & 0.714 \\
\hline 13. Rating my quality of life to be & $-0.42 \pm 0.94$ ( -3 to 2$)$ & $-0.47 \pm 0.95(-4$ to 1$)$ & 0.765 \\
\hline Patient perception of bladder condition & $2.5 \pm 0.6(1-3)$ & $2.4 \pm 0.7(1-3)$ & 0.291 \\
\hline Global response assessment & $5.8 \pm 1.3(2-7)$ & $6.0 \pm 1.0(4-7)$ & 0.447 \\
\hline \multicolumn{4}{|l|}{ Voiding diary parameters } \\
\hline 24-Hour total urine volume $(\mathrm{mL})$ & $1,587.0 \pm 1,025.9(418-3,810)$ & $1,444.2 \pm 460.0(755-2,550)$ & 0.352 \\
\hline Nocturnal urine volume (mL) & $483.7 \pm 301.3(70-1,430)$ & $423.8 \pm 244.8(150-1,173)$ & 0.251 \\
\hline Total number of nightly voids & $1.3 \pm 1.0(4-14)$ & $1.3 \pm 1.1(5-15)$ & 0.991 \\
\hline Maximal bladder capacity (mL) & $317.4 \pm 106.1(120-550)$ & $331.8 \pm 98.6(90-550)$ & 0.461 \\
\hline Differences in maximal bladder capacity $(\mathrm{mL})$ & $-41.3 \pm 96.3(-380$ to 250$)$ & $13.3 \pm 37.9(-60$ to 150$)$ & $<0.001$ \\
\hline Nocturia index & $1.54 \pm 0.87(0.33-5.20)$ & $1.54 \pm 1.01(0.41-6.13)$ & 0.985 \\
\hline Nocturnal polyuria index & $0.37 \pm 0.27(0.08-1.45)$ & $0.29 \pm 0.13(0.11-0.68)$ & $0.028^{*}$ \\
\hline Nocturnal bladder capacity index & $0.48 \pm 0.63(-1.00$ to 2.00$)$ & $0.52 \pm 0.76(-1.00$ to 2.33$)$ & 0.746 \\
\hline
\end{tabular}

Values are presented mean \pm standard deviation (range).

ITT, intention-to-treat; N-QoL, nocturia quality of life.

${ }^{\star} \mathrm{P}<0.05$, statistically significant.

\section{Adverse Events}

A total of 11 patients (8.9\%) showed $\mathrm{AE}$, and none of the patients in either study group showed significant $\mathrm{AE}$. There were 2 cases of dizziness and 1 case of facial flushes in the desmopressin monotherapy group. Each group contained 1 case of peripheral edema and 1 case of voiding difficulties that were not clinically significant. Hyponatremia occurred in 1 case in the desmopressin monotherapy group and in 3 cases in the combination therapy group. Reducing the dose of desmopressin to $0.1 \mathrm{mg}$ im- proved hyponatremia in all cases.

\section{DISCUSSION}

Although behavioral therapy has been shown to be safe and effective in the treatment of patients with NPU [7], a standardized treatment protocol has not yet been widely accepted because nocturia is associated with many factors, including NPU, detrusor overactivity, excessive fluid intakes, evening alcohol or caf- 
feine consumption, inappropriate sleep hygiene, and shortages of daily exercise [2-4]. Nocturia is one of several storage symptoms and it is usually associated with an overactive bladder. Although behavioral modification therapy and medication are initial treatment options for patients with overactive bladders $[5,10]$, behavioral modification therapy has not been widely used in routine clinical practice in patients with nocturia. While both desmopressin and behavioral therapy are effective in patients with NPU $[7,11,12]$, there is little evidence that demonstrates the efficacy of a combination of these treatments. To the best of our knowledge, this is the first investigation to show the efficacy of a combination of desmopressin and SBMP therapy, and to compare the efficacy of desmopressin and the SBMP in combination with desmopressin monotherapy in patients with NPU.

Although the authors performed the SBMP rigorously on the patients, there was no statistically significant difference between the treatment groups with respect to a decline in the number of nocturnal voids, which could be associated with several factors. First, the authors have previously demonstrated the efficacy of the SBMP after reinforcement SBMP education, if the latter was required [5]. However, the SBMP was only performed once in the present study. Second, combination therapy with the SBMP may become less effective when primary treatment with desmopressin is very effective in the treatment of NPU. This suggests that clinicians should be aware of the necessity for repetitive SBMP education for patients with NPU, and that they should consider the patients' baseline characteristics, the severity of the NPU, and drug persistence.

However, desmopressin treatment in combination with the SBMP was more beneficial than desmopressin monotherapy in the management of patients with NPU. The analysis of the NQoL questionnaires showed that changes in the scores for 'productivity the next day' (question 4) and 'concern of disturbing others in the house' (question 8) were more significant in the combination therapy group than in the desmopressin monotherapy group. Additionally, the rate of patients lost to follow up was $10.3 \%(7 / 68)$ in the desmopressin monotherapy group and it was $0 \%(0 / 56)$ in the combination therapy group, which means that the SBMP increased the study completion rate and/or the persistence of desmopressin in patients with nocturia. These results imply that the SBMP significantly affected the daily lives of the patients with NPU and that the patients started to take an active interest in their oral intakes and urine production.

The analysis of the bladder diary parameters suggested that the results from the present study were attributable to increases in the MBC and decreases in the NPI. The baseline MBC was higher in the desmopressin monotherapy group than in the combination therapy group. While there was no difference between the groups with respect to the $\mathrm{MBC}$ at the final outpatient visits, the study findings demonstrated differences in the MBC between the first and the last outpatient clinic visits, which would mean the comparative results of increase or decrease in the MBC between the groups. A previous study investigated the efficacy and safety of desmopressin in patients with mixed nocturia [12] and it showed reductions in the mean number of nocturnal voids, NUV, NPI, and NBCI. The authors of this study also explained that the effect of desmopressin on patients with mixed nocturia was mainly attributable to improvements in the MBC. This suggests that behavioral therapy and the resultant decrease in the number of nocturnal voids increases the efficiency of bladder emptying during sleep.

There are several limitations to the present study that are described next. First, the number of patients who failed the screening assessments was high (38\%; 76/200 patients). Second, the mean number of nocturnal voids was low, which means that the additional effect of the SBMP would have been greater in patients with severe nocturia. Third, we did not perform a urodynamic study to differentiate the mixed type of NPU with detrusor overactivity [10]. Fourth, the starting dose of desmopressin was 0.2 $\mathrm{mg}$ in the present study, which might help to explain why combining desmopressin treatment with the SBMP did not show any significant additional beneficial effects. However, the authors thought that it was better to choose a conservative methodology to determine whether SBMP treatment combined with desmopressin therapy had a beneficial effect on patients compared with desmopressin monotherapy. Fifth, our previous investigation showed that education with 1 SBMP was sufficient for $78.5 \%$ of patients and that reinforcement education with SBMP was helpful for an additional $7.6 \%$ of patients with nocturia [7]. Reinforcement SBMP might have shown a significant beneficial effect in the group that received combination therapy compared with the group that received desmopressin therapy alone; however, we considered that the satisfaction rate from 1 SBMP was not low, and that we needed to simplify the study design to determine the effect of desmopressin and SBMP therapy in combination compared with desmopressin administered alone.

In conclusion, desmopressin monotherapy and the combination of desmopressin with the SBMP reduced the number of nocturnal voids. Combination therapy did not have any addi- 
tional benefits in relation to reducing the number of nocturnal voids in patients with NPU. However, combined desmopressin and SBMP therapy was associated with an increase in the MBC and a decrease in the NPI when it was administered to patients with NPU. Furthermore, combination therapy increased the study completion rate and the persistence of desmopressin in patients with NPU.

\section{ACKNOWLEDGEMENTS}

The authors acknowledge the contribution of Sohee Oh, $\mathrm{PhD}$ (Department of Medical Statistics, SMG-SNU Boramae Medical Center) to the interpretation of the statistical analyses.

\section{REFERENCES}

1. Tikkinen KA, Johnson TM 2nd, Tammela TL, Sintonen H, Haukka J, Huhtala $\mathrm{H}$, et al. Nocturia frequency, bother, and quality of life: how often is too often? A population-based study in Finland. Eur Urol 2010;57:488-96.

2. Soda T, Masui K, Okuno H, Terai A, Ogawa O, Yoshimura K. Efficacy of nondrug lifestyle measures for the treatment of nocturia. J Urol 2010;184:1000-4.

3. Swithinbank L, Hashim H, Abrams P. The effect of fluid intake on urinary symptoms in women. J Urol 2005;174:187-9.

4. Hashim H, Abrams P. How should patients with an overactive bladder manipulate their fluid intake? BJU Int 2008;102:62-6.

5. Bartley JM, Blum ES, Sirls LT, Peters KM. Understanding clinic options for overactive bladder. Curr Urol Rep 2013;14:541-8.

6. Griebling TL. Re: Efficacy of nondrug lifestyle measures for the treatment of nocturia. Editorial comment. J Urol 2010;184:1004; author reply 1004.

7. Cho SY, Lee SL, Kim IS, Koo DH, Kim HJ, Oh SJ. Short-term effects of systematized behavioral modification program for nocturia: a prospective study. Neurourol Urodyn 2012;31:64-8.

8. van Kerrebroeck P, Rezapour M, Cortesse A, Thuroff J, Riis A, Norgaard JP. Desmopressin in the treatment of nocturia: a double-blind, placebo-controlled study. Eur Urol 2007;52:221-9.

9. McKown S, Abraham L, Coyne K, Gawlicki M, Piault E, Vats V. Linguistic validation of the N-QOL (ICIQ), OAB-q (ICIQ), PPBC, OAB-S and ICIQ-MLUTSsex questionnaires in 16 languages. Int $\mathrm{J}$ Clin Pract 2010;64:1643-52.

10. Lee HE, Cho SY, Lee S, Kim M, Oh SJ. Short-term effects of a systematized bladder training program for idiopathic overactive bladder: a prospective study. Int Neurourol J 2013;17:11-7.

11. Kang DI, Kim HM, Oh SY, Yoon JH, Kim HM, Min KS. Short term effect and safety of antidiuretic hormone in the patients with nocturia. Int Neurourol J 2010;14:227-31.

12. Lee HW, Choo MS, Lee JG, Park CH, Paick JS, Lee JZ, et al. Desmopressin is an effective treatment for mixed nocturia with nocturnal polyuria and decreased nocturnal bladder capacity. J Korean Med Sci 2010;25:1792-7. 